with *Anodopetalum and Gaultheria hispida were also abundant as under shrubs, from 2-3000'.

On the crest of the plateau everything had been burnt out, including the Government hut, the tents which replaced it being pitched on burnt wood and ashes. Some distance away a couple of lakelets set in open dwarf forest, which had escaped the flames, duplicated the charm of the summit plateau of Mt Field West. The charred forms of *Athrotaxis selaginoides and *Richea pandanifolia showed that open dwarf forest had also generally prevailed over the plateau, which with several minor rock masses had been swept clear. The highest points, the Hartz Peak with the fine Lake Hartz at its base and the Pinnacle, are both pyramidal masses of broken rock, about $500^{\prime}$ high; the Great Lake, bedded in cliffs and what remained of the forest, lies at the foot of the latter and is quite the most beautiful of the many lakes which form such a striking feature of the Hartz summit plateau.

During the short time spent on this mountain, the atmosphere was so thick with smoke from the many forest fires raging throughout the surrounding country, that it was not only impossible to get any view, but it was difficult to see for any distance on the summit plateau itself.

(To be continued.) 


\title{
ÜBER DIE ENTWICKLUNG DER GESELLSCHAFTS- MORPHOLOGIE
}

\author{
Mitteilungen aus dem Geobotanischen Institut Rübel in Zürich
}

Von EDUARD RÜBEL (Zürich, SchẉEIZ).

Die Lehre von den Pflanzengesellschaften, die Pflanzensoziologie, ist eine relativ neue Wissenschaft, sie hat wohl bei Humboldt schon Anklänge, doch tritt sie erst bei Grisebach, bei Sendtner, Lorenz und besonders bei Kerner selbständig auf und findet ihre Ausgestaltung bei Hult, 1881, Schröter, 1892, und Warming, 1895. Die Pflanzengesellschaft ist an den Standort gebunden, der Standort steht mit ihr in ursächlichem Zusammenhang, der Standort (habitat) ist das Haus, der Oikos, der Pflanzengesellschaft. Es ist daher nicht zu verwundern, dass die Pflanzensoziologie aus der ökologischen Pflanzengeographie hervorgegangen ist, ja bis in die neuste Zeit als ein Teil dieser ökologischen Pflanzengeographie empfunden wird. Die Pflanzensoziologie hat sich aber so mächtig entwickelt, dass sie über diesen Rahmen hinausgewachsen ist. Neben dem Ökologischen, das der Soziologie doppelt anhängt als Forschungsproblem und als eine der wichtigsten Ursachen des Gegenstandes, der Pflanzengesellschaft, fordern auch die anderen biologischen Forschungsprobleme dringend ihre Ausgestaltung. Vor allem stellte sich die Raumfrage nach der Verteilung der Pflanzengesellschaften auf der Erde, es bildete sich eine chorologische Soziologie heraus, ferner eine genetische Soziologie, indem die Veränderungen der Pflanzengesellschaften, ihre Entwicklung verfolgt wurde in der Sukzessionslehre. Diese drei Zweige haben sich schon ziemlich selbständig gemacht. Wie wir bei den Einzelpflanzen als grosse Probleme eine Morphologie und eine Systematik haben, so muss logisch wie praktisch auch bei der Forschung, bei der eine Gemeinschaft, die Pflanzengesellschaft, statt der Pflanzenart den Forschungsgegenstand bildet, sich eine Morphologie und eine Systematik entwickeln. Dem ist auch so; nur werden diese Teile bisher nicht selbständig behandelt, sondern die Beobachtungen, die ihnen angehören, eingeflochten. Ich möchte nun versuchen, die Gesellschafts-Morphologie nach ihren Anfängen, ihrer Entwicklung, kurz nach ihrer Geschichte herauszuschälen.

Fängt man an, eine Pflanze zu untersuchen, so beschreibt man erst ihr Äusseres, ihre Gestalt; so sieht der Stengel aus, so das Blatt, so die Blüte; die Blätter sind als Rosetten vereinigt, oder am Stengel verteilt, die Blüten 

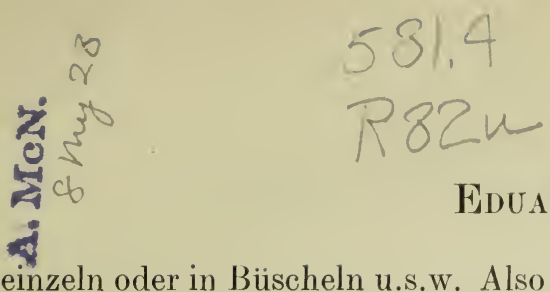

Eduard Rübeis

einzeln oder in Büscheln u.s.w. Also zur Untersuchung werden morphologische Merkmale beschrieben. Die Morphologie hat von jeher eine grosse Rolle bei der Untersuchung der Pflanzen gespielt. Ganz gleich müssen wir bei der Soziologie vorgehen. Was bei der Einzelpflanze die Stellung der Organe, der Blätter, der Bliiten, das vereinzelte oder gedrängte Vorkommen dieser Organe ist, das ist bei der Gesellschaft die Stellung der Arten in der Gesellschaft, das soziale oder vereinzelte Vorkommem, die Mengenverhältnisse der Arten, ihre Konstanz, ihre Treue. Die Morphologie muss also die Gesellschaft qualitativ und quantitativ untersuchen; die Gestalt ist bedingt durch die vorkommenden Arten und ihre Lebensform, durch ihre Mengenverhältnisse, durch ihre Konstanzverhältnisse, durch ihre Treueverhältnisse. In den Untersuchungsmethoden finden wir den Faden der. Gesellschafts-Morphologie. Willdenow, Humboldt, Grisebach, die Väter der Pflanzengesellschaften, gründeten diese auf die Physiognomie. Dies war vortrefflich, aber gefährlich. Man kann die Physiognomie durch bequeme oberflächliche Beschreibung etwa schildern, es ist dies im Laufe des Jahrhunderts ausgiebig geschehen, und es wurde dadurch die ganze physiognomische Betrachtung in Verruf gebracht; man kann die Physiognomie aber auch eingehend als Ausdruck der Gestalt der Gesellschaft studieren, dazu braucht es die Betrachtung der Mengenverhältnisse der Arten, ihrer Verteilung u.s.w., aber in besonderem Masse das Studium der Lebensformen; so wird die Physiognomie ein wesentlicher Bestandteil der Morphologie der Gesellschaft.

Um die Geschichte der Morphologie verfolgen zu können, müssen wir die Fragen, um die es sich handelt, zusammenzustellen versuchen. Morphologische Fragen an die Gesellschaft seien folgende gestellt:

1. Was für eine Bedeutung kommt jeder Art durch Gestalt oder Zahl der Individuen $\mathrm{zu}$ ?

Mengenverhältnisse.

(a) In wieviel Exemplaren in einem Bestand kommt die Art vor?

(b) In welchem Grade herrscht die Art im Bestande?

Abundanz s. str.

(c) In welcher Häufungsweise tritt die Art auf?

Dominanz und Deckungsgrad.

2. Wie regelmässig zeigt sich die Art in verschiedenen Aufnahmen derselben Pflanzengesellschaft?

Konstanz.

3. Wie eng ist die Art an bestimmte Gesellschaften gebunden?

Gesellschaftstreue.

4. Welche Lebensformen treten in der Gesellschaft auf und zu was für Gruppen vereinigen sich diese?

Lebensformen und Schichten (Synusien).

Die Unterscheidung dieser verschiedenen Fragen hat sich naturgemäss erst in neuerer Zeit auseinandergeschält. Früher wurden einige davon mehr oder weniger klar voneinander getrennt, öfter aber nicht auseinandergehalten, im Gegenteil noch vermischt mit der forschungshistorisch älteren, nicht soziologischen Frage nach der geographischen Verbreitung der einzelnen Art. 
In bunter Vermischung wurden für die verschiedensten Dinge die Ausdrücke "häufig," "verbreitet," "zerstreut" u.s.w., gebraucht. Neben der geographischen Einzelverbreitung wurde seit Schouw und Heer auf die Mengenverhältnisse Rücksicht genommen und aus dem allgemeinen Ausdruck "verbreitet" herausgeschält. Es spielt da schon das Berücksichtigen der noch nicht ausdrücklich genannten Pflanzengesellschaft mit, indem von der gewöhnlichen geographischen Verbreitung die Verbreitung innerhalb der betrachteten Gesellschaft, also das Mengenverhältnis abzweigte. Im Laufe der Zeit dachte der eine Forscher bei seinen Bezeichnungen mehr nur an die geographische Verbreitung, der andere schon mit an die soziologische. Dieselben Ausdrücke schliessen bald mehr, bald weniger ein, es entstehen Divergenzen, sodass man die Vegetation verschiedener Länder nach den Literaturangaben meist nicht ohne weiteres vergleichen kann. Aber auch nachdem sich durch das Abtrennen der autochorologischen Verbreitung die Lage etwas geklärt hatte, blieben die Fragen nach Abundanz und Konstanz fast stets gemischt und sind es grossenteils heute noch. Sie können auch nicht bei allen Arbeiten vollständig getrennt werden; bei eingehenden Studien wohl, und dort sollte es auch stets geschehen, bei mehr skizzenhaften Gesellschaftsdarstellungen hingegen lässt sich die synthetische Konstanz nicht klar ausführlich abstrahieren, da Einzelbestand und Bestandestypus meist nicht mehr reinlich getrennt erscheinen. Bei solchen Vegetationsaufnahmen wird meist statt Abundanz- und Konstanzbestimmungen ein Mittelweg eingeschlagen, um ein anschauliches Bild ohne zu grossen Zeitaufwand zu erhalten. Statt vieler Einzelbestände werden die als zur gleichen Gesellschaft gehörig geschätzten Bestände zusammen aufgenommen, was auf der Gesellschaft verbreitet vorkommt als konstant taxiert. So diese floristischen Fragen an die Gestalt der Pflanzengesel]schaft.

Ähnliche Umwege machen auch die ökologischen Gestaltsfragen nach den Lebensformen und deren Gruppierungen in der Pflanzengesellschaft. Bald treten sie rein autökologisch auf, bald als Physiognomie der Pflanzengesellschaft mit historischen und phylogenetischen Komponenten vermischt, bald mit formalen Grössenmerkmalen zu Schichten gruppiert u.s.w.

Wir wollen nun in der Geschichte der morphologischen Soziologie diese beiden Fragenkomplexe, den floristischen und den lebensformlichen, wenn man so sagen darf, verfolgen.

Humboldt $1807^{1}$ erkennt aus der zahllosen Menge der Gewächse eine Reihe von "Grundgestalten oder physiognomischen Abteilungen," auf die man wahrscheinlich alle übrigen Gestalten zurückführen könne, er begnüge sich, 17 derselben aufzuzählen. Es sind dies:

1. Bananenform

3. Form der baumartigen Farrenkräuter

2. Palmenform

4. Aloe-Form

1 Al. v. Humboldt. Ideen zu einer Geographie der Pflanzen nebst einem Naturgemälde der Tropenländer. Tübingen und Paris, 1807. 
5. Pothosform

6. Form der Nadelhölzer

7. Form der Orchideen

8. Mimosenform

9. Malvenform

10. Rebenform

11. Lilienform
12. Cactusform

13. Casuarinenform

14. Gras- und Schilf-Form

15. Form der Laubmoose

16. Form der Blätterflechten

17. Form der Hutschwämme

Diese physiognomischen Abteilungen hat später Grisebach vermehrt. Die Vermehrung brachte aber keine Verbesserung, sondern es entstanden eine Menge Formen, die auf Sippensystematik gegründet waren. Die P'hysiognomie enthält eben zwei Gruppen von Merkmalen: epharmonische oder Anpassungsmerkmale, die für die Lebensformen wie für die Soziologie besonders wichtig sind, und konstitutionelle Merkmale, die in der ökologischen Lebensform nicht viel zu suchen haben. Die Betonung vieler systematisch-konstitutioneller Merkmale hat zeitweise die Physiognomik auf ein totes Geleise geführt.

Unterdessen wird bereits den Mengenverhältnissen Beachtung geschenkt. Schouw $1823^{1}$ spricht von plantae sociales, plantae gregariae, plantae solitariae.

Viel ausführlicher geht Heer $1835^{2}$ vor. Er charakterisiert seine Höhenstufen durch die Anzahl der Arten in Lebensformengruppen, die durch die "Grösse der Individuen" unterschieden sind, also durch Schichten. Er schreibt (S. 78): "Man teilt bekanntlich die Pflanzen hinsichtlich der Grösse der Individuen in Kräuter, Halbgesträuche, Gesträuche und Bäume. In welchem Verhältnisse diese in den verschiedenen Regionen zueinander stehen, zeigt uns folgende Übersicht:

$\begin{array}{lll}\text { Die montane Region hat } \\ \text { die subalpine } & , & , \\ \text { die alpine } & , & , \\ \text { die subnivale } & , & , \\ \text { die nivale } & , & ,\end{array}$

Bäume
21
6
1
0
0

Gestri

$\begin{array}{ccc}\text { striuche } & \text { Halbgestriuche } & \text { Kräuter } \\ 33 & 22 & 479 \\ 11 & 19 & 323 \\ 6 & 21 & 284 \\ 0 & 15 & 201 \\ 0 & 0 & 12^{\prime \prime}\end{array}$

Daneben geht er in der floristischen Charakterisierung von Standorten, wir dürfen schon sagen Pflanzengesellschaften, sehr weit. Zum ersten Mal treffen wir auf die scharfe Unterscheidung von Abundanzverhältnissen und Geselligkeitsverhältnissen. Er erfindet für beide Arten Zahlenskalen und zwar die Zahlen 1-10. Es sei wörtlich zitiert (S. 144): "Durch die Zahlen, welche in den Randkolonnen stehen, suchte ich eine Idee zu geben, durch wieviele Individuen jede Art repräsentiert sei und wie dieselben verteilt sind.

1 Joakim Frederik Schouw. Grundzüge einer allgemeinen Pflanzengeographie. Berlin, 1823.

2 Oswald Heer. "Die Vegetationsverhältnisse des südöstlichen Teils des Kantons Glarus; ein Versuch, die pflanzengeographischen Erscheinungen der Alpen aus klimatologischen und Bodenverhältnissen abzuleiten." Fröbels und Heers Mitteilungen aus dem Gebiete der theoretischen Erdkunde, 1, 3. Zürich, 1835. 
Mit der ersten Zahl nämlich suchte ich auszudrücken, ob eine Form in vielen oder wenigen Individuen sich entfaltet habe. So zeigt z.B. 1 an, dass diese Art eine unbedeutende Rolle hinsichtlich der Individuenzahl spiele, 10 dagegen, dass sie ein hervorstechendes Moment in der Pflanzendecke bilde. Die zweite Zahl gibt an, ob die Art vereinzelt dastehe oder aber gruppenweise die Erde bekleide. Mit 1: 10 will ich daher gesagt wissen, dass eine Pflanze sehr selten sei, wo sie aber einmal vorkomme, in Masse die Erde bekleide; mit 10: 1 aber, dass sie überall sich finde (natürlich nur auf der vorn angegebenen Lokalität), aber nur ganz vereinzelt ins Kleid der Erde eingewoben sei."

Alle die Angaben über Abundanz und Geselligkeit gibt Heer leider nicht im Text bei der Besprechung der Pflanzengesellschaft, für die er den Ausdruck "Lokalität" gebraucht, sondern erst in der sippensystematisch geordneten Florenliste. Von jeder Art gibt er dort an, in welchen Pflanzengesellschaften und in welchen Höhenstufen sie vorkommt und welches ihre Abundanz und Geselligkeit in jeder Gesellschaft ist und dies noch für jede Höhenstufe gesondert. Ein Beispiel möge dies erläutern. Wir finden für Soldanella alpina folgende Angaben:

Soldanella alpina kommt im Sernftal (Kanton Glarus) auf Weiden und in Schneetälchen vor; auf Weiden in der montanen, subalpinen, alpinen und subnivalen Stufe (genannt regio), in Schneetälchen in der alpinen und subnivalen Stufe; in der montanen Weide $1: 3$, also sehr selten aber in kleinen Gruppen; auf subalpinen Weiden $3: 4$, also mässig häufig und truppweise, auf alpinen Weiden $4: 5$, also häufiger und geselliger, auf subnivalen $3: 4$, also wieder weniger häufig und truppweise; in Schneetälchen kommt sie vor: in alpinen Schneetälchen 5:8, also ziemlich häufig und in grossen Heerden, in subnivalen Schneetälchen 4:6, also etwas weniger häufig und in weniger grossen Scharen, immerhin in grösseren als auf irgend einer Weide. Diese Angaben sind von einer Reichhaltigkeit, wie sie von Florenlisten sonst kaum je erreicht wird. Es liegen die Materialien vor, um eine moderne Beschreibung der Pflanzengesellschaften zu geben, aber diese Materialien sind nicht dazu gruppiert worden.

Auf rein floristischem Gebiet hatte als erster Dumont D'Urville $1825^{1}$ Skalen von Zahlen angewandt. Er schätzte die Prozentzahlen der Verbreitung der Pflanzen auf den Falklandinseln (Malwinen) und schätzte an jeder Fundstelle die Art nach ihrer Massenhaftigkeit wiederum in Prozentzahlen. Durch Multiplizieren der beiden Prozentzahlen erhielt er eine Zahl, die er die wirkliche Verbreitung nannte. Obwohl dies gar nichts mit Gesellschaftslehre zu tun hat, könnte das den jungen Heer, falls er D'Urvilles Publikation gekannt haben sollte, auf den Gedanken gebracht haben, Mengenverhältniszahlen für die Pflanzen eines "Standorts" zu verwenden.

1 Dumont D'Urville. "Flore des Malouines." Mém. Soc. Linn. de Paris, 4, 598, 1825; in extenso zitiert in Briquєt. "Les méthodes statistiques applicables aux recherches de floristique." Bull. de l'herbier Boissier, 1, 4, 1893. 
Thurmann in Pruntrut im Berner Jura $1849^{1}$ übernimmt die beiden Heerschen Skalen, aber da er nicht wie Heer an Pflanzengesellschaften, sondern nur an die Gegend als ganzes denkt, werden die Zahlen Verbreitungszahlen und Mengenverhältnisse.

Für die Mengenverhältnisse benutzt auch Lecoq $1844^{2}$ die Zahlen $1: 10$; diese 10 Zahlen fasst er dann noch in vier Wortausdrücke zusammen: $10=$ espèces dominantes, $9-6=$ espèces essentielles, $5-3=$ espèces accessoires, $2-1=$ espèces accidentelles. Im speziellen Teil benutzt er aber weder die Zahlen noch die Ausdrücke. Auch Sendtner $1854^{3}$ benutzt eine Skala für die Dichtigkeit des Vorkommens: $\kappa^{1}$ in Unzahl, $\kappa^{2}$ in Menge, $\kappa^{3}$ in Gesellschaft, $\kappa^{4}$ in Spärlichkeit, $\kappa^{5}$ in Einzelheit. Daneben verwendet er eine ähnliche Skala für die floristische Verbreitung.

Mit Kerner $1863^{4}$ findet die Morphologie nach Lebensformen eine Ausgestaltung, einen grossen Fortschritt. Auch er stellt eine Reihe von "Grundformen" auf, die nach der Physiognomie gewählt sind, doch empfindet er sie wirklich ökologisch, sodass die meisten Formen auf epharmonischen Merkmalen aufgebaut sind, daher als Lebensformen wirken. Er sucht nicht alle Formen der Erde auf einmal zu umfassen, sondern nur die der "heimischen Pflanzenwelt," welche "etwa folgende" Grundformen ergeben: Bäume, Sträucher, Stauden, Filzpflanzen, Kräuter, Blattpflanzen, Schlinggewächse, Fadenpflanzen, Rohr, Halmgewächse, Schwämme, Krustenpflanzen. In vielfach verschlungenem Formengewirre seien diese Grundformen wieder durch Mittelstufen miteinander verknüpft. Mehrere dieser Grundformen seien für die Physiognomie der Landschaft wichtig, andere erscheinen trotz Häufigkeit nicht selbständig und unabhängig, sondern als Beiwerk. Kerner unterscheidet aber nicht nur diese Grundformen, sondern geht noch einen grossen Schritt weiter in der Morphologie der Pflanzengesellschaften. Es sei dies wegen der grossen Wichtigkeit wörtlich zitiert (S. 11):

"Die grösste Wichtigkeit für die Landschaft erlangen die Grundformen natürlich dann, wenn sie ein geselliges Wachstum zeigen und sich zu ganzen Pflanzenmassen von gleichem Ausdruck ineinander schlingen und aneinanderreihen. Man bezeichnet dann solche von einer und derselben Grundform gebildete Pflanzenmassen mit dem Namen Bestände und mag diese wieder, je nachdem sie als vollständig geschlossene Gewebe erscheinen, oder stellenweise durch Felsboden, nackte Erde oder Wasser in ihrem Zusammenhange unterbrochen werden, als Dickichte und Lückichte unterscheiden. Auch unterscheiden wir weiterhin die Bestände nach ihren Grundformen und bezeichnen

1 Jules Thurmann. Essai de Phytostatique, appliqué à la chaîne du Jura et aux contrées voisines, ou étude de la dispersion des plantes vasculaires envisagée principalement quant à l'influence des roches soujacentes. Berne, 1849.

2 Henri Lecoq. Traité des plantes fourragères ou Flore des prairies naturelles de la France. Paris, 1844.

3 Otto Sendtner. Die Vegetationsverhältnisse Südbayerns nach den Grundsätzen der Pflanzengeographie und mit Bezugnahme auf Landeskultur. München, 1854.

4 Anton v. Kerner. Das Pflanzenleben der Donauländer. Innsbruck, 1863. 


$\begin{array}{cc}\text { einen } & \text { Bestand a } \\ , & , \\ , & , \\ , & , \\ , & , \\ , & , \\ , & , \\ , & , \\ , & , \\ , & , \\ , & ,\end{array}$

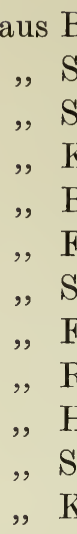

Bäumen

Sträuchern Stauden

Kräutern

Blattpflanzen

Filzpflanzen

Schlingpflanzen, Geschlinge,

Fadenpflanzen , Gefäde,

Röhren

Halmpflanzen

Schwämmen

K als Gehölz,

„, Gesträuch,

,, Gestäude,

,, Gekräut,

„, Geblätt,

Gefilz,

, Geröhr,

, Gehälm,

,, Geschwämm,

, Gekruste.

Von diesen Beständen bedeckt nur selten einer für sich allein den Boden; gewöhnlich findet eine gegenseitige Durchdringung statt und fast immer bemerken wir mehrere Bestände in Schichten übereinander entwickelt, sodass z.B. über einem Gefilz von Moosen, welches als unterste Schichte den Boden bekleidet, das Geblätt von Farnen als zweite Schichte emporstrebt und dann als dritte Schichte die auf schlanken Säulen getragenen dunkeln Kronen eines Fichtengehölzes ihr düsteres Schattendach wölben. Diese Verkettungen von Beständen sind es, welche man Pflanzenformationen genannt hat und deren Studium von den Botanikern unbegreiflicherweise bis in die neueste Zeit so gänzlich vernachlässigt wurde."

Also die Gestalt der "Formation" setzt sich aus ökologisch empfundenen Teilen, genannt "Bestände," die oft als "Schichten" erscheinen, zusammen, und diese Bestände gründen sich auf Grundformen. Die eine Hauptfrage der Gesellschaftsmorphologie nach den Lebensformen und deren Gruppierung erhält hier eine so eingehende Würdigung und fruchtbare Ausgestaltung, auf der nur zu wenig weitergebaut wurde. Dieser Begriff der Kernerschen "Bestände" als ökologischer Teile der Pflanzengesellschaften ist sehr wichtig. Er wird unter verschiedenen Namen auch weiterhin benutzt, aber oft mit den ganzen Pflanzengesellschaften verwechselt. Es kommt dies daher, dass mancher dieser "Bestände" sowohl als untergeordneter Teil einer Pflanzengesellschaft vorkommt als auch in ähnlichem Umfang als dominierend vorkommen kann. Wir kennen z.B. eine selbständige Calluna-Heide einerseits, anderseits Calluna-Bestände im Wald; wir haben submerse Wasservegetation als Pflanzengesellschaft wie auch submerse Bestände im Schilfsumpf. Ähnlich wird Warmings "Verein," "Plantesamfund," als selbständige Gesellschaft benutzt, anderseits auch als Teil einer solchen, wie z.B. aus Schröter $1902^{1}$ zu ersehen ist. Dort lesen wir, dass man im Phragmitetum folgendes findet (S. 68): "im Boden die schlammbewohnenden Diatomeen und Schizophyceen (Schizophyceen-Verein Warmings), im Wasser das Plankton (Phytoplankton

1 Garl Schröter und Otto Kirchner. Die Vegetation des Bodensees. Lindau, 1896 und 1902. II. Teil von Garl Schröter, 1902. 
Warming), die freischwimmenden Uferalgen, auf dem Wasser die Wasserlinsen (Hydrochariten-Verein Warmings), im Schlamm wurzelnd die Charen-, Seerosen- und Potamogetonarten (Limnäen-Verein Warmings) mit ihren epiphytischen Algen, auf den Steinen festsitzende Algen und Moose (NereidenVerein Warmings), in die Luft hinausragend das Schilf (SumpfpflanzenVerein Warmings), wieder mit Parasiten und Epiphyten; also in derselben räumlich und standörtlich verbundenen Pflanzengesellschaft sechs ökologische Vereinsklassen Warmings."

Bei Drude finden wir diese Teilgruppen dann als "ökologischer Verein" zur Unterscheidung von der ganzen Pflanzengesellschaft, die nicht nur ökologisch ist; anderwärts als "Genossenschaften," neuerdings bei Gams als "Synusien." Die Unterscheidung von "Schichten" sucht ebenfalls, wenn auch schematisch, diese ökologischen Unterteilungen einer Pflanzengesellschaft zu fassen.

Eine eigentliche Gesellschaftsmorphologie besitzen wir in Hults (1881) Versuch $^{\mathbf{1}}$ einer analytischen Behandlung der Pflanzenformationen. Darin treffen wir sowohl die Lebensformen und deren Gruppierung und Schichten als auch Abundanzbestimmungen. Er stützt sich auf die bisher besprochenen Forscher, sowie auf J. P. Norrlin, der das Studium der Pflanzengesellschaften in Finnland eröffnet hat. Norrlin hatte schon $1870^{2}$ seine Grundformen aufgestellt: Nadelbäume, Laubbäume, Sträucher, Reiser, Gräser, Kräuter, Schlinggewächse, Blattmoose und Flechten. Hult fügt noch die Sphagnum = Weissmoos-Grundform bei. Hult hat sich abei nicht mit den Grundformen begnügt, sondern diese in 43 "Vegetationsformen" aufgelöst, die teilweise epharmonisch, teilweise aber konstitutioneller Natur wie viele Grisebachsche sind. Diese 43 Formen bilden sodann "Bestände," teils reine, nur aus einer Form bestehende, teils gemischte aus mehr Formen einer Schicht, teils Bestandesgruppen aus mehreren Formen mehrerer Schichten.

Beispiele für Einzelbestände:

Baumbestand der Pinusform

Reiserbestand der Ericaform

Gräserbestand der Calamagrostisform

Kräuterbestand der Geraniumform

Flechtenbestand der Cladinaform.

Gemischte Bestände:

Baumbestand der Pinus- und Betulaformen

Gras- und Krautbestand der Chordorrhiza- und Menyanthesformen.

Bestandesgruppen:

Salix-, Milium-, Geraniumformen

Geranium-, Festuca-, Equisetumformen.

1 Ragnar Hult. "Försök till analytisk behandling af växtformationerna." Meddel. af Soc. pro Fauna et Flora fennica, 8, 1881.

2 J. P. Norrlin. "Bidrag till sydöstra Tavastlands flora." Notiser ur Sällskapets pro Fauna et Flora fennica forhandlingar, 11, 1870. 
Hult betont, dass einzelne dieser "Bestände" geeignet sind, selbständige Pflanzengesellschaften zu bilden, andere nicht.

Den Grössenverhältnissen der nordischen Vegetation entsprechend grenzt Hułt folgende sieben Schichten ab:

1. Bodendecke ... ... ... bis $3 \mathrm{~cm}$. Höhe

$2 a$. Niederste Feldschicht ... ... bis $1 \mathrm{dm}$. Höhe

2 b. Mittlere Feldschicht $\ldots \quad \ldots \quad$ bis $3 \mathrm{dm}$. Höhe

2 c. Höchste Feldschicht $\quad . . \quad \ldots \quad$ bis $8 \mathrm{dm}$. Höhe

3. Strauchschicht ... $\quad \ldots \quad \ldots \quad$ bis $2 \mathrm{~m}$. Höhe

4. Niederwaldschicht $\quad \ldots \quad \ldots \quad$ bis $6 \mathrm{~m}$. Höhe

5. Hochwaldschicht $\quad . . \quad \ldots$ darüber

Neben diesen ökologischen Formen geht bei Hult auch die Beachtung der Mengenverhältnisse. Die Skala dazu scheint er schon von seinem Lehrer Norrlin übernommen zu haben. Er verwendet die Zahlen 1-10 und fügt für ganz vereinzelt vorkommende Arten noch zwei weitere Stufen r und rr bei. Für praktische Arbeiten fasst er sie in 5 Stufen zusammen. Genau geschildert zitiert Linkola $1916^{1}$ (S.40) als "Dichtigkeitsskala Norrlins" folgendeımassen :

Deckend oder häufig (ymniga)

10. Grad der Beimischung anderer Arten 1-4

$9 ., \quad, \quad, \quad, \quad, \quad 4-6$

8. " $, \quad, \quad, \quad, \quad 6-7 \frac{1}{2}$

Reichlich (rikliga)

7. Zwischenraum zwischen Individuen der fraglichen Art $2 \frac{1}{2}-15 \mathrm{~cm}$.

$6 . \quad, \quad, \quad, \quad, \quad, \quad, \quad, \quad 15-50 \mathrm{~cm}$.

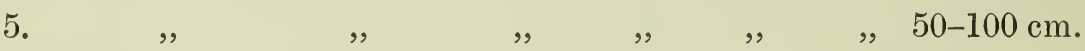

Zerstreut (strödda)

4. Zwischenraum zwischen Individuen der fraglichen Art 1-2 m.

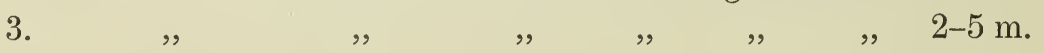

Spärlich (spridda)

2. Zwischenraum zwischen Individuen der fraglichen Art 5-10 m.

1.

. " , " $, \quad, \quad, \quad$, über $10 \mathrm{~m}$.

Vereinzelt (enstaka)

r

rr

Hult hat nun diese Skala, die wir ganz entsprechend bei Heer, Thurmann und Lecoq gefunden haben, zum ersten Mal zur genauen Abschätzung der Mengenverhältnisse innerhalb der Pflanzengesellschaft verwendet, wodurch die Gesellschaftsmorphologie eine grosse Förderung erhielt. Im Prinzip ähnliche Skalen werden fast allerorten gebraucht, so von Drude die Ausdrücke

1 K. Linkola. "Studien über den Einfluss der Kultur auf die Flora in den Gegenden nördlich vom Ladogasee. I. allgemeiner Teil." Acta Soc. pro Fauna et Flora fennica, 45, 1916. 
socialis, copiosus, sparsus, soltarius; von den Fngländern dominant, abundant, frequent, occasional, rare, very rare ${ }^{1}$.

Von Hult an halten sich die Untersuchungen meist auch an einen bestimmten Rang von Gesellschaften, nämlich an die grundlegrende Einheit, die wir heute Assoziation nennen. Im weiteren historischen Verlauf der Morphologie handelt es sich nun fast stets um die Morphologie der Assoziation.

Hult hat für die Charakterisierung der Gesellschaft eine knappe graphische Darstellung verwendet unter Benützung der Mengenverhältnisse, der Grrundformen und Schichten. Ein Viereck wird so zerteilt, dass jede Schicht einen horizontalen Streifen erhält. In senkrechten Kolonnen werden die 5 Häufigkeitsgrade eingetragen, der häufigere jeweilen doppelt so breit wie der vorhergehende. In jeder Schicht wird so weit schraffiert als der vorhandene Häufigkeitsgrad angibt. Hinter die Horizontalstreifen wird die Zahl der Grundform gesetzt, die in jener Schicht herrscht nach der Aufstellung
I Nadelbäume
VI Kräuter
II Laubbäume
VII Schlinggewächse
III Gebüsch
VIII Weissmoose (Sphagna)
IV Reiser (Zwergsträucher)
IX Blattmoose
V Gräser
X Flechten

Ein Beispiel mag dies erläutern:

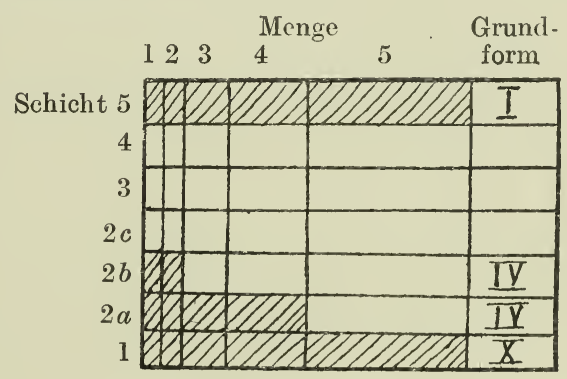

Aus der Figur lesen wir: Geschlossener (M. 5) Nadelholz (I)-Hochwald (Sch. 5), ohne Gesträuch (0 Sch. 4; 0 Sch. 3); spärliche (M. 2), mittelhohe (Sch. 2 b) Zwergstrauchschicht (IV); reichliche (M. 4), niedrige (Sch. 2 a) Zwergstrauchschicht (IV) und deckende (M. 5) Flechten (X)-Bodenschicht (Sch. 1). Hult hat diese Aufnahme am 12. Aug. 1877 in Kittilä in Kemi Lappmark gemacht: Die Hochwaldschicht besteht aus Pinus silvestris mit eingestreuter Picea; in der niedrigen Feldschicht findet sich reichlich Vaccinium vitis idaea, zerstreut Arctostaphylos uva ursi, spärlich Empetrum, Calluna und Festuca ovina; die Sträuchlein ragen zum Teil spärlich in die mittlere Feldschicht hinauf. Die Bodenschicht besteht aus Sterencaulon paschale 5, Cladinen und

1 Erstmals ganz durchgeführt in C. E. Moss, Geographical distribution of vegetation in Somerset: Bath and Bridgwater District. Royal Geographical Society, London, 1906. Für ganz Grossbritannien durchgeführt in dem hervorragenden Werk: A. G. Tansley, Types of British Vegetation. Cambridge, 1911. 
Cetrarien 2, Hylocomium und Polytrichum 3, Linnaea borealis 2; als Epiphyten Baumflechten.

Um zum Ausdruck zu bringen, dass die höheren Schichten mehr Masse darstellen und wichtiger sind, hat Sernander die Figur etwas verändert, d.h. die höheren Schichten brẹiter gezeichnet, was folgendes Beispiel (aus Lagerberg $1914^{1}$, S. 138) veranschaulichen mag:

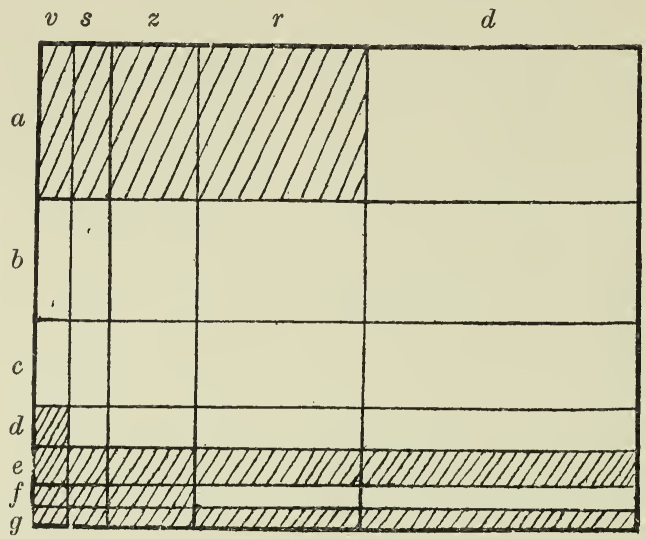

Moosreicher Kiefernwald mit dichter Heidelbeerdecke.

a Hochwald: Kiefer,

$d$ höchste Feldschicht: Fichte,

e mittlere Feldschicht: Vaccinium myrtillus,

$f$ niederste Feldschicht: Vaccinium vitis idaea, Calluna,

$g$ Bodenschicht: Hylocomium parietinum.

In anderer Weise wird die Morphologie behandelt in stebler und Schröter $1892^{2}$ zur Charakterisierung der schweizerischen Wiesentypen. Nach den Mengenverhältnissen werden Hauptarten, Nebenarten und Akzessorische unterschieden. Von da an führt Schröter in der Schweiz die Schätzung der Mengenverhältnisse in den Pflanzengesellschaften nach der Skala 1-10 ein. Merkwürdigerweise stiutzt er sich dabei auf Lecoq und nicht auf seinen Lehrer Heer. Das mag daher kommen, dass Heer diese Morphologie in einer Jugendarbeit ausgearbeitet hatte und seine Studien später eine andere Richtung genommen hatten. Stebler und Schröter führen ihre Untersuchungen nach einer gewichtsanalytischen Methode durch. Sie wählen einen typischen Fleck in der Pflanzengesellschaft aus, um an einem abgemessenen Quadrat die vorkommenden Arten und den genauen Anteil einer jeden zu bestimmen. Als Grösse nahmen sie einen Quadratfuss (der alte schweizerische Fuss hat eine Länge von $30 \mathrm{~cm}$.). Der wurde ausgestochen und jedes Pflan-

1 Torsten Lagerberg. "Markflorans analys på objektiv grund. Meddelanden från Statens Skogsförsöksanstalt, 11." Skogsvördsföreningens Tidskrift, 1914.

2 F. G. Stebler und Carl Schröter. "Versuch einer Übersicht über die Wiesentypen der Schweiz. 10. Abh. der Beiträge zur Kenntnis der Matten und Weiden der Schweiz." Landwirtschaftiches Jahrbuch der Schueiz, 6. Bd. 1892. Bern, 1893. 
zentrieblein herauspräpariert, nach Arten gesondert, das Material jeder Art für sich getrocknet und gewogen. Auf diese Weise erhält man den genauen Anteil der Arten in Gewichtsprozenten am Aufbau des Quadratfusses der betreffenden Pflanzengesellschaft. Damit tritt in die Gesellschaftsmorphologie die Quadratmethode ein, die dafür von grosser Wichtigkeit wurde. Sie wurde später nicht nur gewichtsanalytisch ausgebeutet, sondern erwies sich auch für andere Methoden der Abundanzbestimmung, besonders für Zählmethoden brauchbar und wurde in England und Amerika weit ausgestaltet.

Pound und Clements $1898^{1}$ benutzen zum Auszählen der Abundanz in der Prärie ein Quadrat von $5 \mathrm{~m}$. Seitenlänge. Sie nennen dann $\operatorname{cop}^{1}$ eine Spezies, die in mehr als 200 Exemplaren in diesem Quadrat vorkommt, cop ${ }^{2}$ erhalten Arten mit 150-200 Exemplaren, cop ${ }^{3}$ mit 100-150 Stuick, cop ${ }^{4}$ mit 50-100, subcop ${ }^{1} 15-50$, subcop ${ }^{2} 5-15$, spars mit 0,1-5 Stück.

Eine weitere Ausgestaltung gibt Clements der Quadratzählung $1904^{2}$ und $1905^{2}$. Es werden hauptsächlich Quadrate von $1 \mathrm{~m}$. Seite verwandt, grössere von $2 \mathrm{~m}$. Seitenlänge sind nötig in Gehölzen und bei offener Vegetation. Es erfolgt nicht nur ein Zählen der Individuen, sondern auch ein Kartieren dieser Quadrate. Diese können markiert werden als Dauerquadrate, in denen man die Veränderungen der Vegetation im Laufe der Jahre nachweist. Denselben Dienst leistet auch die Modifikation von Oliver und Tansley $1904^{3}$, welche die Messquadrate zu Rastern vereinigen. Neben den besprochenen Zählquadraten, Kartenquadraten, Dauerquadraten verwendet Clements noch entblösste Quadrate, um Neubesiedelung experimentell zu untersuchen, ferner Linien- und Gürteltranssekte, das sind lineare Querschnitte durch die Vegetation.

Hier seien auch die Jaccardschen Untersuchungen von 1900, 1901, 1902 , 1908, $1914^{4}$ erwähnt. Jaccard stellt sich die Aufgabe, Gesetzmässigkeiten fuir die Artverteilung im Raume zu finden. Er sucht zahlenmässige Verhältnisse zwischen Floren verschiedener Gebiete herzustellen und ferner Verhältnisse zwischen der Zahl der Arten und der Zahl der Gattungen. Je gleichartiger die Verhältnisse von Örtlichkeiten, um so höher wird in der Regel ihr Gemeinschaftskoeffizient sein innerhalb des gleichen Florengebietes. Die Untersuchungen Jaccards beziehen sich nicht auf Pflanzengesellschaften, sie sollen nicht zur Charakterisierung von solchen dienen, wie dies von einzelnen Forschern

1 Roscoe Pound and Frederic E. Clements. The Phytogeography of Nebraska. I, General survey. Lincoln, Neb. 1898. (Second edition, 1900.)

2 Frederic E. Clements. The development and structure of vegetation. Studies in the vegetation of the State III. Lincoln, Neb. 1904. Frederic E. Clements. Research methods in ecology. Lincoln, Neb. 1905.

${ }^{3}$ F. W. Oliver and A. G. Tansley. "Methods of surveying vegetation on a large scale." New Phytologist, 3, 1904.

4 Verschiedene Arbeiten über die Pflanzenverteilung in Flora, Bd. 90, 1902; Bull. Soc. vaud. sc. nat. 38, 1902; Rev. gén. des sc. Paris, 1907; Bull. Soc. vaud. sc. nat. 44, 1908; Rev. gén. bot. 26. Paris, 1914. 
vorausgesetzt worden ist, sondern sie wollen die Gesetzmässigkeiten der Beziehungen der Florenbezirke aufdecken. Sie könnten vielleicht abec auch dazu verwendet werden, Verschiedenheit der Standorte darzustellen, daher die Erwähnung in der Gesellschaftsmorphologie.

Es folgt in den Jahren 1905, 1907 ein grosser Fortschritt in den die Lebensformen betreffenden Fragen durch die Arbeiten von Raunkiär ${ }^{1}$. Er stellt ein Lebensformensystem auf Grund der epharmonischen Eigenschaft der Anpassung an die Überdauerung der ungünstigen Jahreszeit auf. In seiner Absicht liegt die Charakterisierung der Klimate durch den Anteil der einzelnen Lebensformen nach Artenzahl in bestimmten Gebieten. Der Zweck ist nicht auf Gesellschaftsbestimmung gerichtet, aber die aufgestellten Formen lassen sich auch zu diesem Zwecke benutzen, und sie wurden auch von Raunkiär 1909, von Vahl u.a. bald dazu benutzt. Er stellt 30 Lebensformen auf, die er aber meistens zur praktischen Verwendung in 10 Gruppen zusammenzieht. Von einem Gebiet gibt er dann den Prozentsatz an Arten jeder Lebensform an. Dies wird als biologisches Spektrum bezeichnet. Zur vergleichenden Beurteilung stellt er ein Normalspektrum auf, das die Prozentsätze der Arten der ganzen Erde vorstellen soll. Gewisse Modifikationen wurden von Ostenfeld und von Vahl an dem System ausgeführt.

Es folgt nun wiederum ein Wendepunkt für die floristischen Fragen der Gestaltsuntersuchung. Die bisher besprochenen Methoden richteten sich mehr oder weniger an den konkreten einzelnen Bestand, die Einzelsiedelung. Die Pflanzengesellschaft, in erster Linie ihre grundlegende Einheit, die Assoziation, ist naturgemäss eine Abstraktion, dieZusammenfassung der gleichen Bestandesindividuen. Eine Vergesellschaftung mit bestimmter Artenliste tritt in der Regel an vielen Örtlichkeiten mit gleichen Standortsbedingungen auf. Die Synthese aus vielen Einzelsiedelungen ergibt dann die Assoziation. Es ist also nötig, die Ähnlichkeiten der Artenlisten verschiedener Aufnahmen in Beziehung zu bringen. Dies lässt sich schätzungsweise oder dann statistisch durch Zusammenstellung der Artenlisten bewerkstelligen. Eine einzelne Aufnahme ist nicht in allen Bestandteilen das Mittel der Assoziation. Die Artenlisten werden nie ganz identisch ausfallen, eine gewisse Variation findet von einer Stelle zur andern stets statt. Um wieviel Arten dürfen nun zwei Listen sich unterscheiden, um noch zur selben Assoziation gerechnet zu werden? Darüber können all die bisher besprochenen Darstellungen der Mengenverhältnisse nur mangelhaft Auskunft geben. Ein Nebeneinanderstellen der zusammengehörenden Aufnahmen in Tabellenform, aus denen man die Ähnlichkeit herauslesen könnte, hat erstmals Cajander $1903^{2}$ ausgeführt,

${ }^{1}$ Christen Raunkiär. Planterigets Livsformer og deres Betydning for Geografien. Kopenhagen, 1907.

2 A. K. Cajander. "Die Alluvionen des unteren Lena-Tales. I. Abh. der Beiträge zur Kenntnis der Vegetation der Alluvionen des nördlichen Eurasiens." Acta soc. scient. fennicae, 32, 1903. 
aber die Konsequenzen für die Konstanz nicht daraus gezogen. Die klare Unterscheidung zwischen den Mengenverhältnissen der einzelnen Aufnahmen und den Konstanzverhältnissen der Assoziation hat erst Brockmann 19071 herausgearbeitet. Dieses neue morphologische Moment hat sich später so fruchtbar erwiesen und grösseren Ausbau erhalten, dass es hier etwas ausführlicher im Zusammenhang behandelt sei.

Die früheren Methoden der Abundanzprüfung wollen eigentlich zugleich auch herausfinden, was die Konstanten sind. Sie tun es nicht direkt, sondern indirekt nach folgender Überlegung: Die Arten, die in grösster Menge vorkommen, sind dem gegebenen Standort demgemäss am besten angepasst und werden wohl daher für die Assoziation bezeichnend sein. Dies wird in vielen Fällen zutreffen. Es ist aber auch möglich, dass lokale Umstände, besondere lokale Besiedelungsmöglichkeiten, Arten in einem Einzelbestand zum Vorherrschen bringen, die sonst nicht für die Assoziation bezeichnend sind. Durch die Brockmannsche Methode macht man sich erstens von diesen Zufälligkeiten unabhängig, zweitens noch von folgender Tatsache, die sonst verschleiernd wirken kann: Arten, die nicht wählerisch in Bezug auf Standort sind, die man euryözisch nennen kann, da sie verschiedene Standorte besiedeln, können an verschiedenen Örtlichkeiten herrschend werden, ohne dass diese Örtlichkeiten derselben Assoziation angehören, trotzdem sie äusserlich ähnlich aussehen können. Erst die Prüfung der Artenkonstanz ergibt objektiv die Zugehörigkeit der Einzelsiedelungen zum Typus, zur Assoziation. Man mache daher möglichst viele Aufnahmen einer Assoziation. Die Arten, die mindestens in der Hälfte aller Aufnahmen vorkommen, nennt Brockmann die Konstanten, solche die in weniger als der Hälfte aber mindestens in einem Viertel der Aufnahmen vorkommen, die Akzessorischen, die übrig bleibenden nennt er zufällige Beimischungen. Bei der Aufnahme der Einzelsiedelung ist auf Einheitlichkeit des Standorts zu achten. Dass an einem durchfliessenden Bächlein z.B. der Standort völlig anders ist, wird jedem klar sein, aber es kommen auch ganz geringe Veränderungen vor, über deren Zugehörigkeit man verschiedene Auffassungen haben kann, eine engere oder eine weitere. Bei der Konstanzbestimmung nach dieser statistischen Methode wird diese Subjektivität korrigiert. Die allzu breite Auffassung wird die Zahl der zufälligen Beimischungen erhöhen, sie aber als solche erkennen lassen, denn bei der Zusammenstellung der Aufnahmen in Tabellenform fallen schlecht gemachte Aufnahmen, die nicht zur Assoziation gehören, durch ihre heterogene Liste auf; man kann sie ausscheiden, und es zeigt sich klar, was wirklich zusammengehört. Hier korrigiert sich auch von selber der etwas zu viel sagende Satz, dass die Assoziation durch die gesamte Artenliste charakterisiert sei. Hier kann man die Beimischungen ausscheiden, die für die Assoziation nicht massgebend sind; vorbehalten die Treuebestimmungen, von denen

1 Heinrich Brockmann-Jerosch. "Die Flora des Puschlav (Bezirk Bernina, Kanton Graubünden) und ihre Pflanzengesellschaften." Die Pflanzengesellschaften der Schweizeralpen, 1. Leipzig, 1907. 
später die Rede sein wird. Die Konstanzbestimmung führt auch aus dem Wirrwarr der Übergänge heraus. Nicht jeder Fleck Erde trägt eine abgeschlossene Assoziation; es kommen unendlich viele Übergänge vor, die man nicht gleich erkennt; hier machen sie sich durch Fehlen von Konstanten, durch Auftreten und Häufigwerden fremder Beimischungen geltend.

Eine Hauptsache bei der Konstanzstatistik ist, dass man eine genügende Anzahl Aufnahmen macht und diese möglichst gleichmässig auf das Gebiet verteilt. Nimmt man viele Aufnahmen von ganz genäherten Flecken, so können Arten in die Massgebenden hineingeraten, die bei gleichmässiger Berücksichtigung des Gebietes nur Beimischungen sind. Kommt z.B. eine Art in 3 Aufnahmen von 6 vor, so wäre sie noch konstant, 2 weitere Aufnahmen ohne sie verweisen sie in die Reihe der Akzessorischen (3 von 8) und noch zwei weitere ohne sie (also 3 von 10) unter die Zufälligen. 6 Aufnahmen sind also unbedingt zu wenig, um vollwertige Massgebende daraus abzuleiten. Nehmen wir dagegen 30 Aufnahmen und eine Akzessorische darin komme 14 Mal vor: Sollte sie nun in 25 weiteren Aufnahmen auch gar nicht mehr vorkommen, so bleibt sie doch noch Akzessorische, denn 14 ist immer noch mehr als ein Viertel der Aufnahmen: $14>\frac{1}{4}(30+25)=13 \cdot 75$.

Wie man in der morphologischen Systematik der Einzelpflanzen nicht einig ist, wie weit man eine Spezies oder Varietät fassen soll und die Zahl der Spezies von Forscher zu Forscher sehr variiert, so ist es auch bei den Assoziationen. Man kann verschiedener Ansicht sein, wieviele der Massgebenden (Konstante + Akzessorische) wechseln dürfen, dass man noch von derselben Assoziation sprechen darf, oder von einer neuen Assoziation oder nur einer anderen Subassoziation. An der Zahl gemeinsamer Konstanten kann die Verwandtschaft sich nahestehender Gesellschaften beurteilt werden. Eine ausgedehnte Anwendung der Konstanzbestimmung, sei es nach der statistischen Methode oder nach einer andern, z.B. einer schätzungsweisen, scheint mir berufen zu sein, viel Licht in das Wesen der Assoziation und ihrer Unterabteilungen zu bringen. Auch über die Konkurrenzverhältnisse der Arten und die Besiedelungsfähigkeit kann diese Bestimmung viel aussagen. Die von Brockmann für die Fettwiesen des Puschlav erstlich durchgeführte Konstanzbestimmung fand dann eine ausgedehnte Anwendung in den Pflanzengesellschaften des Berninagebietes durch Rübel 1911.

Diese statistische Methode hat die Wichtigkeit der Konstanz so richtig herausgearbeitet. Vorher und nachher wurden, ohne den Namen Konstanz $\mathrm{zu}$ brauchen und das Wesen der Angaben klarzulegen, die konstanten Arten in Gesellschaftsbeschreibungen stets als bedeutend hervorgehoben, als die Pflanzen, aus denen die Gesellschaft besteht, die sie bedingen oder aufbauen. Beim flüchtigen Beobachter waren es dann in Wirklichkeit nur Abundanzzahlen weniger Beobachtungsorte, bei grüdlichen Beobachtern hingegen kann diese bloss geschätzte Konstanz fast so richtig ausfallen wie die nach

"s 1 Eduard Rübel. "Pflanzengeographische Monographie des Berninagebietes." Englers Bot. Jahrb. 47. Bd. 1911 und im Buchhandel, Leipzig, 1912. 